\title{
"KAMBOH": A QUALITATIVE STUDY OF POSTPARTUM CARE IN KUTAI ETHNIC TRIBE, EAST KALIMANTAN, INDONESIA
}

\author{
Annisa Nurrachmawati ${ }^{1}$, Anna Marie Wattie ${ }^{2}$, Mohammad Hakimi ${ }^{3}$ and Adi Utarini ${ }^{4}$ \\ ${ }^{1}$ Department of Biostatistics, Population Study, and Reproductive Health Faculty of Public Health, Mulawarman \\ University, 7511, Samarinda, Kalimantan Timur, Indonesia \\ ${ }^{2}$ Department of Anthropology, Faculty of Cultural Science, Universitas Gadjah Mada, 55281, Bulaksumur, Yogyakarta, \\ Indonesia \\ ${ }^{3}$ Department of Obstetrics and Gynaecology, Faculty of Medicine, Universitas Gadjah Mada, 55281, Bulaksumur, \\ Yogyakarta, Indonesia \\ ${ }^{4}$ Department of Health Policy and Management, Faculty of Medicine, Universitas Gadjah Mada, 55281, Bulaksumur, \\ Yogyakarta, Indonesia
}

Corresponding author: Annisa Nurrachmawati

Email: nasywa_mzi@yahoo.com

\begin{abstract}
Postpartum is recognized as a critical period in many cultures, when societies view mothers as vulnerable. $A$ longitudinal qualitative study of pregnant women in the rural area of Kutai Kartanegara, East Kalimantan, Indonesia was conducted to explore cultural beliefs and practices during the postpartum period. Ethnography approach was performed and the data on the postpartum period are presented. Intensity sampling was used to select informants from Muara Kaman Ulu and Ilir villages, Muara Kaman Sub district, Kutai Kartanegara and in-depth interviews were conducted with 17 Kutai postpartum women, two midwives, and two traditional birth attendants. Data analyses were conducted using the thematic analysis method. The main health problem occurring in the postpartum period is called "kamboh". It is a whole body ache with many symptoms, mainly fever, becoming skinny, with burning sensation that suddenly appears on the legs, itchiness and shivering. In order to prevent it women should undergo dietary and physical activity restrictions for 40 days. Certain foods were perceived as the possible causes of the itchy condition, delayed wound healing and hemorrhage. Following the traditional taboo, going outside the house and working hard were restricted. Close families and traditional birth attendants play important roles to ensure compliance of postpartum women to these restrictions. Understanding of cultural beliefs during the postpartum period is critical to support women. Therefore, health promotion interventions with particular attention to diet and physical activity should be designed by taking into account the local practices.
\end{abstract}

Keywords: postpartum care, culture, beliefs, Kutai ethnic tribe, longitudinal qualitative ethnography

\section{INTRODUCTION}

The postpartum period is a critical time for the recovery of reproduction organs, when various health concerns may occur, ranging from mild to severe physical concerns that include fatigue, loss of appetite, headache, and back pain ${ }^{1}$. The health concerns may also be in the form of mental health disorders such as anxiety and depression ${ }^{2}$. Postpartum is also considered critical from the socio-cultural aspects. Each society has a different understanding of risks in the postpartum period, therefore every culture has a distinctive method in maternal health care. For example, the people of Nepal and Morocco view childbirth as a dirty condition so that the bleeding in postpartum period is considered as an effort to purge the body of contamination and not considered as a lifethreatening condition..$^{3,4}$. In Indonesia, the Alifuru ethnic in Maluku encourages women to increase their meals to speed up the recovery ${ }^{5}$. The cultural context related to pregnancy and childbirth is often considered incompatible with modern medical concepts.

This poses a problem when modern medical interventions seek to shift the influence of cultural elements without trying to better understand more of its practices and values which influence behavior of health care services utilization ${ }^{6}$. Indonesia has rich cultural context and ethnic thribes such as the Kutai thribe in Kalimantan which is known to have a strong cultural belief. This community also has the lowest visit in the health centre for postpartum care. Therefore, the attempts to understand the cultural construction of each culture are required to provide services more suitable to the women's needs. This study aims to explore the culture and behaviors of women during the postpartum period in the Kutai ethnic tribe, Kutai Kartanegara, East Kalimantan in Indonesia.

\section{METHODS}

Study design and sample selection

This research is part of a longitudinal qualitative study with an ethnographic approach aimed to understand the dynamics of decision making in choosing place of birth in the Kutai ethnic tribe. Study was conducted in Muara Kaman Ulu and Muara Kaman Ilir villages, in the area of The Muara Kaman health centre with the highest number of maternal deaths in the province and the lowest 
proportion of women visiting the health centre for postpartum care $(37.6 \%)^{7}$ in the Kutai Kartanegara District (Figure 1). This village is accessible through three hours drive from the city of Tenggarong with poor road conditions. Health facilities vary from integrated health posts, three midwife private practices, and a Community Health Centre.These facilities are easily accessible by motorcycle within 5-10 minutes.

Pregnant women's experiences in deciding the place of birth were explored longitudinally from pregnancy to the postpartum period. Using an intensity sampling, we selected only pregnant women in the first and second trimester out of 23 pregnant women in the first six months of data collection. Selection of women in the first two trimesters enable the study to capture a more intense experience in decision making process in the overall study design. We identified the informants from the health centre record, except for the first trimester where we added a snowball technique sampling due to delayed first antenatal care leading to underestimation of the health centre record. Three pregnant women were excluded due to the following reasons: moving out from the village, miscarriage, and preterm labor.

\section{Data collection and analysis}

Data collection were conducted from April 2015 to April 2016. Recruitment of informants was discontinued when no new information obtained after the third interviews. The first author and one research assistant performed the interviews, using local language or combined with Indonesian. They both have public health background, are fluent with the local language, and lived in the study area. Data reported in this paper came from in-depth interviews in the postpartum phase. To obtain a comprehensive understanding and to improve validity, triangulation of data collection methods was conducted through in-depth interviews with two health center midwives, and two traditional birth attendants (TBA).

After obtaining informants' consent, the entire interview was recorded and transcripts were produced immediately afterward. The data were analyzed using thematic analysis. Codes were generated, organized using Opencode software (version 4.0), and grouped to form categories focusing on perceptions and behaviours during the postpartum period. Informants were assured that data were only used for the research purposes and remained confidential. Ethical clearance for this study was received from the Ethics Committee of Medical and Health Research, Faculty of Medicine, Universitas Gadjah Mada.

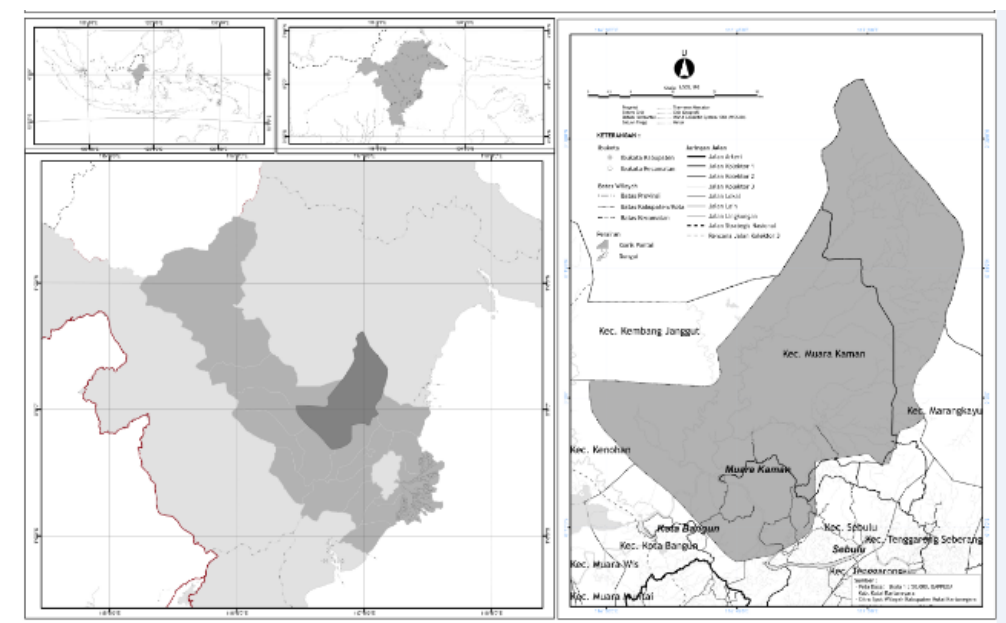

Fig. 1 Map of Indonesia displaying province and district of study site

\section{RESULTS}

\section{Informants' Characteristics}

Seventeen pregnant women were interviewed, consisting of two primigravid and 15 multigravid pregnant women. Their age ranged from 19 to 37 years old. Only three women were working as teachers at various levels of education and the rests are housewives. The highest education level of most women was junior high school and five women got married at a young age, i.e. seventeen years.
Kamboh: A typical condition in the postpartum period

The main health problem experienced by postpartum women in Kutai is known as kamboh. In general, kamboh describes the pain throughout the body experienced by women after giving birth with various symptoms such as: fever, shivering, dizziness, itchiness and a burning sensation in the legs that can suddenly appear. These symptoms can appear instantly after restriction was violated or later during or after the postpartum period. Symptoms may only last for a while if the women immediately seek treatment with traditional medicine. Some women recover with modern medical treatment. 
There are different kinds of kamboh; each has different symptoms. In kamboh bentan, the body becomes thin, with wrinkled, dry and blackened skin. If the woman steps on the floor which is exposed to the sun without wearing any footwear, the soles of the feet will remain hot even though it is no longer stepping on the floor (kamboh langat). If the woman's feet aches like being punctured with a sharp object when stepping in the rainwater, this is known as kamboh hujan. Kamboh makan occurs when the woman's body feels hot but without having a fever after consuming prohibited foods. The severe form of Kamboh is called kamboh bekerajaman, when it is accompanied by shivers at night either with or without a fever until fatal. As described by a TBA "After childbirth the mother can experience kamboh. The body turns skinny, always drooling, and it can cause death, after continuing to shiver at night" (I1, aged 63).

Behavior Restrictions in the Postpartum Period A number of behavior restrictions for postpartum women exist, such as the necessity to use footwear when going outside the house to avoid stepping directly in the rainwater or on a sunexposed floor to avoid kamboh hujan or kamboh langat. One informant stated " kamboh feels like your feet being punctured by needle, you cannot step on rainwater on wooden floor, you must wear sandals" (13, aged 36). Violating this restriction can cause a burning sensation to the feet and the heat will not disappear even if the mother is no longer stepping on the floor.

The head of postpartum women should also not be exposed to the sun, because the sun is believed to cause dizziness, which is one of the kamboh symptoms. This restriction limits the postpartum women from going outside the house. If they have to go out, they must not hit their feet on hard objects, because it is believed to cause bleeding: "I'm afraid of kamboh. If I am going outside, I cannot move freely as usual, I must be careful to not hit my foot" (12, aged 24).

Postpartum women and their infants are forbidden to leave the house or travel for $\mathbf{4 0}$ days. This restriction can be broken in case of urgent needs, i.e. presence of health problems on the mother or the baby that require immediate action at the health care. For illustration, one informant preferred to still stay at home when the baby was referred to a hospital due to medical condition. Work restrictions or limited physical activities exist. Thus, assistance from the husband and family members is needed for domestic work in the first seven days after giving birth. A late morning shower may cause the mother's skin to dry, while sleeping without a pillow is believed to increase the white blood to the eye and creates a blurred vision. Sexual intercourse is also prohibited until postpartum ends as marked by the postpartum bath carried out by the women according to the Islam religion. If these restrictions are violated, the mothers will suffer from kamboh bentan.

\section{Food Restriction in the Postpartum Period}

Consumption of food and beverages are controlled. Postpartum women are not allowed to consume hot beverages and meals, which induce oral thrush to occur in the baby through breastfeeding. Dietary restrictions are applied to the type of fish with many bones particularly large bones, such as belida and pahat fish, along with some types of tubers such as yams and eggplant, because it causes itchiness in the genitalia. Postpartum women are only allowed to drink water in limited quantities after eating. Too much drinking is believed to cause the uterus becomeing wet and will not recover as quickly as before.

The variaty of food is limited to vegetables, bananas, green beans, which is perceived to increase breastmilk volume, and certain large fish such as smoked snakehead and baung fish. Sources of vegetable protein such as tempeh and tofu are acceptable, with only a little oil. Sources of animal protein other than fish are prohibited, as expressed: "A soup of young banana or green bean only, we cannot eat chicken or beef, because they can cause bleeding" (16, aged 32$)$.

\section{Prevention and Treatment of Kamboh}

The prevention of kamboh is carried out through restrictions of certain foods or behavior. For example, to prevent bleeding due to hitting a foot, postpartum women wears black bracelet threads that have been read out of prayers on the thumb of their right and left foot. These bracelets are usually made and paired from the beginning of the postpartum period by the TBAs as a reminder that postpartum women have to be careful when walking. Similarly, if the mothers want to travel, before leaving the house, they should use foot wear and head gear to prevent kamboh langat. Other kamboh preventions include drinking herbal potions, made from some leaves, turmeric and tamarind. This herb is routinely taken once a day, usually before meals. Postpartum women take this herb for three days after delivery or up to 40 or even 100 days. Additional to strengthen the body and to eliminate fatigue, they also apply pupur kamboh, a kind of body mask made from fruit skin and herbs. Another traditional treatment for postpartum women is abdominal massage for three days after birth to recover to womb's position. If the massage is combined with the herbal drink, it is believed to flow the dirty blood out.

When the postpartum women violate the restrictions, the treatment of kamboh varies according to symptoms. If the women are shivering with or without fever, the treatment is to drink herbs made from the skin of durian, ginger and lemongrass taken once to three times a day until healed. For legs with burning sensation, mothers soak their feet in the water 
with metal objects such as knife that have been exposed to the sun. If the mothers accidentally consume prohibited foods resulting in burning sensations to the body or bleeding, the TBA suggested "eat cat fish, take the bone and wipe it to the head and the body" (11, aged 63).

Interviews with the midwives indicated that cultural beliefs during postpartum may cause less visits to the health center. If the women have Kamboh, they tend to resort to the TBAs first, before seeing a midwife. Therefore, the midwives proactively conduct home visits for medical examinations, family planning and problems in breastfeeding. These visits are free of charge, but due to limited human resources and time, not all postpartum women were visited. The midwives also emphasized that postpartum women do not need to refrain from eating and to have abdominal massage. Even though postpartum women understood this advice, they chose to adhere to the restrictions, as expressed: "The midwives said that it's okay, there are no food restrictions, they even encourage us to eat nutritional items like sea fish, but we are too afraid" (17, aged 27). Nevertheless, we found two informants (multipara with higher education and working as teachers) who no longer practice the restrictions and prevention of Kamboh: "No I didn't do food restriction nor abdominal massage. The doctor said it wasn't necessary" (18, aged 28).

\section{DISCUSSION}

The results show that postpartum women from Kutai ethnic tribe in Kutai Kartanegara still hold strong cultural beliefs that affect their behaviour. This result is consistent with other studies. Duration of postpartum for 40 days as well as the restrictions of food and behavior were also found in the Malaysian culture ${ }^{8}$, food restrictions in Thailand culture 9 , and in Chinese, postpartum women are prohibited from consuming cold food or drink and recommended to stay at home. ${ }^{10}$ These previous studies showed similarities in terms of avoiding heavy work, prohibition of traveling, the suggestion of sitting at home, as well as a number of food restrictions.

Family members and TBAs are the primary source of information regarding local beliefs and practices. Adherence of postpartum women to these local practices can be seen as an effort to respect senior family members, and to maintain social acceptance. In this way, postpartum women continue receiving support from their families and communities in their role as parents. The study conducted by Kaewsarn ${ }^{11}$ in Thailand also showed that in the atmoshere where cultural practices still have a strong influence, postpartum women strive to obey them to avoid conflict.

From a health perspective, several cultural practices can be potentially harmful, i.e. restrictions of food and not traveling for 40 days.
Although this study did not specifically measure food intake of postpartum women, restriction of foods may limit the source of protein and other nutrients that are needed for recovery. Adequate protein intake is necessary for the wound healing of childbirth and breastfeeding. The WHO guidelines for health in the period of postpartum recommends mothers to actually increase the intake of calories up to $10-20 \%$ in the daily diet during breastfeeding ${ }^{12}$.

Another cultural practices that may have a negative effect is water intake limitation. An adequate water intake is needed during lactation since breastmilk production requires adequate maternal body water. Regardless of not enough evidence showing that extra fluids for breastfeeding mothers actually increase milk production, sufficient fluid intake is necessary to maintain their health ${ }^{13}$. The European Food Safety Authority has estimated the total water needed in lactating women is about 600 to $700 \mathrm{~mL} / \mathrm{d}^{14}$ to compensate for loss of water during milk production. The Indonesian Ministry of Health also sets recommendations for lactating women to increase total water intake by $800 \mathrm{~mL} /$ day during the first six months exclusively breastfeeding and by $650 \mathrm{~mL} /$ day after six months ${ }^{15}$.

Some behavioral precautions such as restriction of physical activity may also have an unfavorable impact on health. According to the WHO guideline, all postpartum women should be encouraged to do light exercises with enough time to rest ${ }^{12}$. Light exercise was associated with the mother's body fitness, postpartum weight retention, and improvement of mother's mental health ${ }^{16-17}$. This restriction is also perceived to induce less postpartum visits to health centers, leading to less interaction with the midwives, and less opportunity to get health messages. The WHO guideline encourages midwives or other health workers to conduct home visits for women who cannot visit the health facilities, to enable them to obtain services and receive information ${ }^{12}$. This, however, was not practiced regularly in the study area.

Even though tradition is still strongly practiced by the women, few women with higher education no longer perform traditional care. This was consistent with $\mathrm{Liu}^{10}$ who showed that higher education women received postpartum home visit and attended postpartum courses, hence, tend not to practice traditional care". However, attention is still needed in the implementation of traditional postpartum care, because this may constrain the women from accessing postpartum care. Simply ignoring the existence and values of these cultural practices may not result in effective postpartum care as well. Studies have shown that interventions focusing on increasing access and improving quality without respecting local views related to pregnancy and childbirth proved to have less optimal positive impact ${ }^{18-19}$. 
Therefore, understanding of the cultural context is critical because the community's perception of the needs and the cause of health problems can be a predictor in determining the behavior in health services' utilization ${ }^{20}$.

The use of ethnography approach in this study enables detailed description on cultures surrounding postpartum care in specific tribal communities such as Kutai. Although cultural practices differ among localities, the messages about the need to improve cultural sensitivity remains for a health program to be successfully implemented. Despite their important roles to support postpartum women, the views of men and grandmothers were confirming the women's views, hence not presented in this paper.

\section{CONCLUSIONS}

In conclusion, this study provided a better understanding of the cultural postpartum practices that are strongly believed amongst Kutai women. Understanding these values in a specific ethnic group would promote culturally appropriate care to support postpartum women. Based on our results, we recommend that health promotion programs should be designed by taking into account the cultural sensitivity, focusing on healthy diet and physical activity, with pregnant women, their family members and the TBAs as the main target audiences. This strategy may increase community acceptance toward modern health care interventions to improve women's health in the postparum period. Emphasis on the importance of postpartum care should also be given since early pregnancy.

\section{ACKNOWLEDGEMENTS}

We acknowledged the Ministry of Research, Technology and Education for the financial support for this research through the Graduate Program Scholarship. We thanked the study participants, health centers, and a special thanks to Tantim Filial for assisting with data collection. The authors declare that they have no competing interests.

\section{REFERENCES}

1. Lee, Ji Yeon \& Hwang JY. A study on postpartum symptoms and their related factors in Korea. Taiwanese $\mathrm{J}$ Obstet Gynecol 2015; 54(4): 355-63.

2. Klainin P. \& Arthur DG. Postpartum depression in Asian cultures: A literature review. Int. J. Nurs. Stud 2009; 46(10): 1355-73.

3. Obermeyer, CM. Pluralism and pragmatism: knowledge and practice of birth in Morocco. Med Antrophol Q 2000; 14(2): 180-201.
4. Matsuyama A. \& Moji K. Perception of bleeding as a danger sign during pregnancy, delivery and the postpartum period in rural Nepal. Qual Health Res 2008; 18(2): 196-208.

5. Permana M, Friskarini K, Litaay SC. Maternal and Child Health Ethnographic Series: Ethnic Alifuru Seram, Maluku, Centre for Health Policy and Community Empowerment, National Institute of Health Research and Development, Indonesia: Ministry of Health; 2012. 100p.

6. Inhorn, MC. Arab American, Africa American, and infertility: barrier to reproduction and medical care. Fertil Steril 2016; 85(4): 844-52.

7. Health District Office of Kutai Kertanegara. Health Profil Report of Kutai Kertanegara

Kutai Kartanegara District Health Office 2016.

8. Fadzil F, Shamsuddin K, Wan Puteh SE. Traditional postpartum practices among Malaysian mothers: a review. J Altern Complement Med 2015; 22(7): 503-8.

9. Thurakal $S$, Lunderberg $P$, Chaladthanyagid $\mathrm{K}$, et al. Traditional postpartum practices and food consumption among women in rural Thailand. Journal of Public Health 2007; 37: 178-190.

10. Liu N, Mao L, Sun X, et al. Postpartum practices of puerperal women and their influencing factors in three regions of Hubei, China. BMC Public Health 2006; 6:274-281.

11. Kaewsarn, P., Moyle, W., Creedy, D. Traditional postpartum practices among Thai women. J Adv Nurs 2003; 41(4): 358-366.

12. World Health Organisation. Guidelines on postnatal care for mothers and newborns: Geneva: World Health Organisation. 2013. 72p. Available from http://www.who.int/maternal_child_ad olescent/documents/postnatal-carerecommendations (accessed 21 Feb 2017).

13. Ndikom $\mathrm{CM}^{1}$, Fawole B, Ilesanmi RE. Extra fluids for breastfeeding mothers for increasing milk production. Cochrane Database Syst Rev 2014; 11(6): CD008758

14. EFSA Panel on Dietetic Products, Nutrition, and Allergies (NDA). Scientific 
Opinion on Dietary reference values for water. EFSA Journal 2010; 8(3):1459.

15. Ministry of Health of the Republic of Indonesia. Recommended Balance Nutritional Intake for Indonesian Population. Indonesia: Ministry of Health; 2013. 99p. Available from gizi.depkes.go.id.

16. Martin J, Mac Donalds-Wicks L, Hure A, et al. Reducing postpartum weight retention and improving breastfeeding outcomes in overweight women: a pilot randomized controlled trial. Nutrients 2015; 7(3): 1464-79.

17. Blum J, Beaudoin C, Caton-Lemos L. Physical activity pattern and maternal well-being. Matern Child Health J 2004; 8(3): 163-69.

18. Myer L \& Harrison A. Why do women seek antenatal care late? Perspective from rural South Africa. J Midwifery Womens Health 2003; 48(4): 268-72.

19. Berry N. Kaqchikel midwives, home births, and emergency obstetric referrals in Guatemala: Contextualizing the choice to stay home. Soc Sci Med 2006; 62(8): 1958-69.

20. Ensor T\& Cooper S. Overcoming barriers to health service access: influencing the demand side. Health Policy and Plan 2004; 19(2): 69-79. 